\title{
Prognostic factors in patients admitted to an urban teaching hospital with COVID-19 infection
}

Donogh Maguire ${ }^{1 *} \mathbb{D}$, Marylynne Woods ${ }^{2 \dagger}$, Conor Richards ${ }^{2 \dagger}$, Ross Dolan ${ }^{3}$, Jesse Wilson Veitch², Wei M. J. Sim², Olivia E. H. Kemmett' ${ }^{2}$, David C. Milton², Sophie L. W. Randall², Ly D. Bui' ${ }^{2}$ Nicola Goldmann², Allan Cameron ${ }^{4}$, Barry Laird ${ }^{5,6}$, Dinesh Talwar ${ }^{7}$, lan Godber ${ }^{8}$, Alan Davidson ${ }^{1}$ and Donald C. McMillan ${ }^{3}$

\begin{abstract}
Background: Severe COVID-19 infection results in a systemic inflammatory response (SIRS). This SIRS response shares similarities to the changes observed during the peri-operative period that are recognised to be associated with the development of multiple organ failure.

Methods: Electronic patient records for patients who were admitted to an urban teaching hospital during the initial 7-week period of the COVID-19 pandemic in Glasgow, U.K. (17th March 2020_1st May 2020) were examined for routine clinical, laboratory and clinical outcome data. Age, sex, BMI and documented evidence of COVID-19 infection at time of discharge or death certification were considered minimal criteria for inclusion.

Results: Of the 224 patients who fulfilled the criteria for inclusion, 52 (23\%) had died at 30-days following admission. COVID-19 related respiratory failure (75\%) and multiorgan failure (12\%) were the commonest causes of death recorded. Age $\geq 70$ years $(p<0.001)$, past medical history of cognitive impairment $(p \leq 0.001)$, previous delirium $(p<0.001)$, clinical frailty score $>3(p<0.001)$, hypertension $(p<0.05)$, heart failure $(p<0.01)$, national early warning score (NEWS) $>4(p<0.01)$, positive CXR $(p<0.01)$, and subsequent positive COVID-19 swab $(p \leq 0.001)$ were associated with 30-day mortality. CRP $>80 \mathrm{mg} / \mathrm{L}(\mathrm{p}<0.05)$, albumin $<35 \mathrm{~g} / \mathrm{L}(\mathrm{p}<0.05)$, peri-operative Glasgow Prognostic Score (poGPS) $(p<0.05)$, lymphocytes $<1.510^{9} / \mathrm{l}(p<0.05)$, neutrophil lymphocyte ratio $(p \leq 0.001)$, haematocrit $(<0.40 \mathrm{~L} / \mathrm{L}$ (male) $/<0.37 \mathrm{~L} / \mathrm{L}($ female $))(p \leq 0.01)$, urea $>7.5 \mathrm{mmol} / \mathrm{L}(p<0.001)$, creatinine $>130 \mathrm{mmol} / \mathrm{L}(p<0.05)$ and elevated urea: albumin ratio $(<0.001)$ were also associated with 30 -day mortality.

On multivariate analysis, age $\geq 70$ years (O.R. 3.9, 95\% C.I. 1.4-8.2, $p<0.001$ ), past medical history of heart failure (O.R. 3.3, 95\% C.I. 1.2-19.3, p<0.05), NEWS > 4 (O.R. 2.4, 95\% C.I. 1.1-4.4, p<0.05), positive initial CXR (O.R. 0.4, 95\% C.I. 0.2$0.9, \mathrm{p}<0.05)$ and poGPS (O.R. 2.3, 95\% C.I. 1.1-4.4, $\mathrm{p}<0.05)$ remained independently associated with 30-day mortality. Among those patients who tested PCR COVID-19 positive $(n=122)$, age $\geq 70$ years (O.R. 4.7, 95\% C.I. 2.0-11.3, $p<0.001$ ), past medical history of heart failure (O.R. 4.4, 95\% C.I. 1.2-20.5, p<0.05) and poGPS (O.R. 2.4, 95\% C.I. 1.1-5.1, $\mathrm{p}<0.05)$ remained independently associated with 30-days mortality.
\end{abstract}

\footnotetext{
*Correspondence: Donogh.Maguire@glasgow.ac.uk

${ }^{\dagger}$ Marylynne Woods and Conor Richards contributed equally to this work

${ }^{1}$ Emergency Medicine Department, Glasgow Royal Infirmary, 84 Castle

Street, Glasgow G4 OSF, UK

Full list of author information is available at the end of the article
}

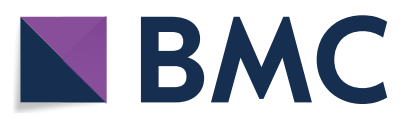

(c) The Author(s) 2020. This article is licensed under a Creative Commons Attribution 4.0 International License, which permits use, sharing, adaptation, distribution and reproduction in any medium or format, as long as you give appropriate credit to the original author(s) and the source, provide a link to the Creative Commons licence, and indicate if changes were made. The images or other third party material in this article are included in the article's Creative Commons licence, unless indicated otherwise in a credit line to the material. If material is not included in the article's Creative Commons licence and your intended use is not permitted by statutory regulation or exceeds the permitted use, you will need to obtain permission directly from the copyright holder. To view a copy of this licence, visit http://creativeco mmons.org/licenses/by/4.0/. The Creative Commons Public Domain Dedication waiver (http://creativecommons.org/publicdomain/ zero/1.0/) applies to the data made available in this article, unless otherwise stated in a credit line to the data. 
Conclusion: Age $\geq 70$ years and severe systemic inflammation as measured by the peri-operative Glasgow Prognostic Score are independently associated with 30-day mortality among patients admitted to hospital with COVID-19 infection.

Keywords: COVID-19, Systemic inflammatory response (SIRS), C-reactive protein (CRP), Albumin, Peri-operative glasgow prognostic score (poGPS), Neutrophil lymphocyte ratio (NLR), 30-day mortality, Host inflammatory response

\section{Background}

As of 27th May 2020, approximately 5.7 million people worldwide are known to have been infected with COVID-19 coronavirus and more than 350,000 have died [1]. The severity of this viral disease for an individual is associated with a widespread perturbation of immune, physiological and metabolic parameters [2, 3]. These whole body changes could be considered characteristic of a systemic inflammatory response to tissue injury and it has been long recognised that a large and ongoing systemic inflammatory response is associated with the development of multiple organ failure and infective disease $[4,5]$.

One of the cardinal signs of severe COVID-19 infection is a marked systemic inflammatory response [2]. This response bears striking similarity to the systemic inflammatory response experienced by patients undergoing major elective surgical resections for cancer [6, 7]. Indeed, the systemic inflammatory response and the associated metabolic stress has been most well characterised in major elective surgery, where the relationship between the magnitude of the post-operative systemic inflammatory response and the development of postoperative complications is now well recognised, as is the effect of patient comorbidity on this relationship [8, 9]. Such work has informed therapeutic manoeuvres including minimally invasive surgery, pre-operative optimisation (e.g. anaesthesia, nutrition and steroids) and enhanced recovery protocols.

The aim of the present study was to examine whether routinely collected clinicopathological characteristics of patients with COVID-19 on admission were informative on the immune and metabolic stress experienced by patients with COVID-19 and whether such characteristics were informative on subsequent outcome.

\section{Patients and methods}

Electronic patient records for patients who attended the Emergency Department (ED) and Acute Assessment Unit (AAU) at Glasgow Royal Infirmary (GRI), Glasgow, U.K., during the initial 7-week period of the COVID-19 pandemic in Glasgow city (17th March 2020-1st May 2020) were examined for routine clinical, laboratory and clinical outcome data. GRI is a university teaching hospital that serves an urban population with a high burden of socio-economic deprivation and offers the full spectrum of adult acute receiving specialties to patients over 16 years old. In line with NHS policy, this study was approved by the NHS Greater Glasgow and Clyde Caldicott guardian. The study protocol (GN20AE307) was approved by the North West England-Preston research ethics committee $(20 / \mathrm{NW} / 0336)$ and registered with clinicaltrials.gov (NCT04484545).

Patients displaying clinical signs or reporting symptoms consistent with possible COVID-19 infection (as defined by Health Protection Scotland) [10] at the time of presentation to ED and AAU were assessed for inclusion in the study. Patients who were reported by a board certified radiologist to have radiological changes characteristic of COVID-19 infection reported on chest X-ray (CXR) or CT thorax, were assessed for inclusion in the study. Patients who were admitted with other conditions and tested polymerase chain reaction (PCR) positive following admission were also included in the analysis. SARS-CoV-2 PCR testing was performed on all patients included in the sample, however only 122 of the 264 patients who satisfied HPS criteria for clinical diagnosis of SARS-CoV-2 and were admitted to hospital, subsequently had the diagnosis confirmed with positive SARSCoV-2 PCR test. Age, sex, BMI and documented (clinical, radiological or PCR) evidence of COVID-19 infection at time of discharge or death certification were considered minimal criteria for inclusion.

As per routine clinical practice in the Emergency Department and Acute Assessment Area at GRI, patients were scored on the National Early Warning Score (NEWS) at presentation to triage. NEWS is a validated score of severity of physiological derangement that allocates a score $(0-3)$ to six clinical parameters (pulse rate, blood pressure, respiratory rate, oxygen saturations, requirement for supplemental oxygen and level of responsiveness (alert (A), responding to verbal (V), painful (P) stimuli and unresponsive (U) (AVPU scale)) [11]. NEWS determines the triage category and level of immediate treatment that is required at the time of presentation, and the interval to re-administering the NEWS scoring tool according to the score achieved (i.e. the severity of physiological derangement). NEWS $>4$ and $>7$ are considered to indicate moderately severe and severe physiological derangement respectively. 
Table 1 Calculation of the Neutrophil Lymphocyte Ratio (NLR)

\begin{tabular}{lcl}
\hline Neutrophil Lymphocyte Ratio (NLR): & Ratio & SIRS severity \\
\hline Neutrophil count: lymphocyte count & $<3$ & Mild \\
Neutrophil count: lymphocyte count & $3-5$ & Moderate \\
Neutrophil count: lymphocyte count & $>5$ & Severe \\
\hline
\end{tabular}

Table 2 Peri-operative Glasgow Prognostic Score (poGPS)

\begin{tabular}{lll}
\hline $\begin{array}{l}\text { peri-operative Glasgow Prognostic Score } \\
\text { (poGPS) }\end{array}$ & Score & SIRS severity \\
\hline $\begin{array}{l}\text { C-reactive protein } \leq 150 \mathrm{mg} / \mathrm{l} \text { and Albu- } \\
\text { min } \geq 25 \mathrm{~g} / \mathrm{l}\end{array}$ & 0 & Mild \\
$\begin{array}{c}\text { C-reactive protein }>150 \mathrm{mg} / \mathrm{l} \text { and Albu- } \\
\text { min } \geq 25 \mathrm{~g} / \mathrm{l}\end{array}$ & 1 & Moderate \\
$\begin{array}{l}\text { C-reactive protein } \leq 150 \mathrm{mg} / \mathrm{l} \text { and Albu- } \\
\text { min }<25 \mathrm{~g} / \mathrm{l}\end{array}$ & 1 & Moderate \\
$\begin{array}{c}\text { C-reactive protein }>150 \mathrm{mg} / \mathrm{l} \text { and Albu- } \\
\text { min }<25 \mathrm{~g} / \mathrm{l}\end{array}$ & 2 & Severe \\
\hline
\end{tabular}

Age was grouped as less than 40 years, $40-49$ years, 50-59 years, 60-69 years, 70-79 years and 80 years and older. Age categories were further simplified to $</ \geq 70$ years (see Tables $3,4,5$ ). Social deprivation was defined by the Scottish Indices of Multiple Deprivation 2019 based on individual home postcode. Ethnicity was classified as White, Mixed, Asian, Black, or other ethnic group. Frailty was assessed using the Clinical Frailty Scale (CFS) $[12,13]$.

Admission serum C-reactive protein (CRP), albumin and differential blood cell counts were categorised using local reference intervals. Neutrophil/lymphocyte ratio (NLR) and the peri-operative Glasgow Prognostic Score (poGPS) were calculated as outlined in Tables 1, $2[6,14$, 15]. The neutrophil lymphocyte ratio (NLR) is a validated prognostic scoring system that has been used in a variety of clinical settings. It utilises two components of the differential white cell count that are routinely measured in patients admitted to the general hospital setting. However, studies utilising the NLR in sepsis and peri-operative prognostic scores have used a variety of thresholds, making inter-study extrapolation of results difficult. For this study, thresholds of NLR $\leq 3,>3-<5$ and $\geq 5$ have been chosen, indicating mild, moderate and severe systemic inflammatory response respectively [16].

\section{Statistical analysis}

Autobiographical data, clinicopathological data and haematological/biochemical results were presented as categorical variables. Categorical variables were analysed using $\chi^{2}$ test for linear-by-linear association, or $\chi^{2}$ test for 2-by-2 tables.

Associations between autobiographical data, clinicopathological characteristics, haematological/biochemical results and survival were analysed using univariate and a multivariate backward conditional approach. A $p<0.05$ was applied to inclusion at each step in the multivariate analysis.

A convenience sampling strategy was adopted based on the patients admitted during the study period; therefore a formal sample size calculation was not performed. Missing data were excluded from analysis on a variable-byvariable basis. Two-tailed $p$ values $<0.05$ were considered statistically significant. Statistical analysis was performed using SPSS software version 25.0. (SPSS Inc., Chicago, IL, USA).

\section{Results}

Of the 359 patients who attended Glasgow Royal Infirmary and satisfied HPS criteria for categorising as a possible COVID -19 related presentation, 241 patients fulfilled the criteria for inclusion with age, sex, BMI and documented evidence of COVID-19 infection at discharge or death certification. Seventeen patients were re-admitted and these were excluded from the analysis at second admission leaving 224 patients to be included in the analysis. The clinicopathological characteristics at presentation are shown in Table 3 . The majority of patients were $\geq 70$ years old (88\%), male (55\%), were not obese $(57 \%)$ and were socioeconomically deprived (SE groups 1 and 2, 64\%). The majority of patients were living independently in their own home (85\%) and of white Scottish (93\%) ethnicity. The majority of patients did not have comorbid disease including hypertension (60\%), heart failure (90\%) or type 2 diabetes (77\%) and were not frail (54\%). The median BMI was $29.0 \mathrm{~kg} / \mathrm{m}^{2}$, with $33 \%$ of individuals having a BMI of less than $26 \mathrm{~kg} /$ $\mathrm{m}^{2}$, and $25 \%$ exceeding a BMI of $35.0 \mathrm{~kg} / \mathrm{m}^{2}$.

The median temperature of patients was $37.0{ }^{\circ} \mathrm{C}$ (IQR $\left.36.3-38.0{ }^{\circ} \mathrm{C}\right)$. The majority of patients had a temperature $<37.5{ }^{\circ} \mathrm{C}(65 \%)$ and $14 \%$ of patients had a temperature $<36{ }^{\circ} \mathrm{C}$. On admission the majority of patients had moderately severe or severe physiological derangement (NEWS score $>4$ ) (60\%) and had radiological changes characteristic of COVID-19 infection reported on chest $\mathrm{X}$-ray $(59 \%)$.

Of the laboratory analysis at presentation, the majority of patients had evidence of a systemic inflammatory response as evidenced by an elevated CRP $>80 \mathrm{mg} / \mathrm{L}$ (51\%) and NLR (80\%). The majority of patients had bilirubin $(88 \%)$, alkaline phosphatase (91\%), AST (54\%), ALT (79\%), glucose (53\%), urea (67\%), creatinine (90\%), sodium (86\%), potassium (80\%), MCV (84\%) and platelets $(80 \%)$ within the laboratory reference range. 
Table 3 Univariate analysis of clinicopathological characteristics of patients admitted with symptoms of COVID-19 $(\mathrm{n}=\mathbf{2 2 4})$

\begin{tabular}{|c|c|c|c|}
\hline & Alive $(n=172)$ & Dead $(n=52)$ & p-value \\
\hline $\operatorname{Age}(</ \geq 70$ years $)$ & $124 / 48$ & $19 / 33$ & $<0.001$ \\
\hline Sex (male/female) & $91 / 81$ & $33 / 19$ & 0.181 \\
\hline $\operatorname{BMI}\left(<20 ; \geq 20-29 ; \geq 30 \mathrm{~kg} / \mathrm{m}^{2}\right)$ & $13 / 85 / 74$ & $4 / 25 / 23$ & 0.867 \\
\hline SIMD (1 (most)-6 (least) deprived) & $81 / 31 / 17 / 23 / 1 / 19$ & $24 / 4 / 7 / 11 / 0 / 6$ & 0.685 \\
\hline Ethnicity $(1-5)$ & $159 / 0 / 7 / 2 / 3$ & $50 / 0 / 0 / 0 / 2$ & 0.774 \\
\hline Living circumstances (0-3) & $151 / 11 / 7 / 3$ & $39 / 2 / 8 / 3$ & 0.008 \\
\hline \multicolumn{4}{|l|}{ Past Medical History } \\
\hline Hypertension (y/n) & $63 / 109$ & $28 / 24$ & 0.027 \\
\hline Heart failure $(y / n)$ & $12 / 160$ & $11 / 41$ & 0.003 \\
\hline $\mathrm{T1DM}(\mathrm{y} / \mathrm{n})$ & $2 / 170$ & $0 / 52$ & 0.436 \\
\hline T2DM (y/n) & $37 / 135$ & $15 / 37$ & 0.273 \\
\hline Chronic renal failure $(y / n)$ & $18 / 154$ & $10 / 42$ & 0.095 \\
\hline Cognitive impairment $(\mathrm{y} / \mathrm{n})$ & $16 / 156$ & $14 / 38$ & 0.001 \\
\hline Previous delirium $(y / n)$ & $6 / 166$ & $10 / 40$ & $<0.001$ \\
\hline Frailty score $(\leq />3)$ & $107 / 65$ & $14 / 38$ & $<0.001$ \\
\hline $\operatorname{COPD}(\mathrm{y} / \mathrm{n})$ & 29/143 & $12 / 40$ & 0.311 \\
\hline Smoker (never/ex/active) & $82 / 70 / 20$ & $24 / 27 / 1$ & 0.428 \\
\hline Alcohol excess $(y / n)$ & $22 / 150$ & $9 / 43$ & 0.410 \\
\hline Liver disease $(y / n)$ & $15 / 157$ & $4 / 48$ & 0.816 \\
\hline Hep C (never/previous/active) & $167 / 2 / 2$ & $51 / 1 / 0$ & 0.650 \\
\hline Active cancer $(\mathrm{y} / \mathrm{n})$ & $6 / 166$ & $3 / 49$ & 0.464 \\
\hline Asthma $(y / n)$ & $42 / 130$ & $4 / 48$ & 0.009 \\
\hline Surgery < 1 yr (y/n) & $17 / 154$ & $4 / 44$ & 0.277 \\
\hline Cancer resection (y/n) & $1 / 171$ & $0 / 52$ & 0.582 \\
\hline \multicolumn{4}{|l|}{ Diagnostic criteria } \\
\hline PCR positive/Clinical Dx/Radiological Dx & $74 / 7 / 91$ & $37 / 1 / 14$ & 0.001 \\
\hline PCR negative/indeterminate/positive & $43 / 42 / 83$ & $4 / 9 / 39$ & 0.001 \\
\hline CXR negative/positive & $63 / 107$ & $30 / 21$ & 0.006 \\
\hline \multicolumn{4}{|l|}{ Physiology at presentation } \\
\hline NEWS $(\leq />4)$ & $75 / 96$ & $12 / 39$ & 0.009 \\
\hline Delirium (y/n) & $14 / 158$ & $12 / 36$ & 0.001 \\
\hline \multicolumn{4}{|l|}{ Laboratory results at presentation } \\
\hline $\mathrm{CRP}(</ \geq 150 \mathrm{mg} / \mathrm{L})$ & $134 / 37$ & $34 / 18$ & 0.058 \\
\hline Albumin $(\geq /<35 \mathrm{~g} / \mathrm{L})$ & $82 / 87$ & $15 / 35$ & 0.021 \\
\hline $\operatorname{poGPS}(0 / 1 / 2)$ & $130 / 35 / 3$ & $32 / 15 / 3$ & 0.032 \\
\hline $\operatorname{WCC}\left(<4.5 / \geq 4.5-\leq 11.0 />11.0 \times 10^{9} / \mathrm{L}\right)$ & 23/118/30 & $7 / 34 / 11$ & 0.750 \\
\hline Neutrophils $\left(</ \geq 7.5 \times 10^{9} / \mathrm{L}\right)$ & $132 / 39$ & $34 / 18$ & 0.088 \\
\hline Lymphocytes $\left(\geq /<1.5 \times 10^{9} / L\right)$ & $52 / 118$ & $7 / 45$ & 0.015 \\
\hline $\operatorname{NLR}(<3 / 3-5 / \geq 5)$ & $39 / 46 / 85$ & $5 / 9 / 38$ & 0.004 \\
\hline $\mathrm{Hb}(\geq /<12.0 \mathrm{~g} / \mathrm{dL})$ & $143 / 28$ & $38 / 13$ & 0.142 \\
\hline $\mathrm{MCV}(>/ \leq 99 \mathrm{fl})$ & $149 / 21$ & $40 / 11$ & 0.102 \\
\hline Hct (male $\geq /<0.40$ ) (female $\geq /<0.37$ ) L/L & $126 / 45$ & $29 / 23$ & 0.014 \\
\hline Platelets $\left(\geq /<150 \times 10^{9}\right)$ & $140 / 30$ & $40 / 12$ & 0.383 \\
\hline Sodium $(<133 / \geq 133-\leq 146 />146 \mathrm{mmol} / \mathrm{L})$ & $17 / 153 / 2$ & $9 / 40 / 3$ & 0.013 \\
\hline Potassium $(<3.5 / \geq 3.5-\leq 5.5 />5.5 \mathrm{mmol} / \mathrm{L})$ & $13 / 142 / 2$ & $5 / 36 / 0$ & 0.822 \\
\hline $\mathrm{Mg}(\geq /<0.75 \mathrm{mmol} / \mathrm{L})$ & $33 / 60$ & $8 / 28$ & 0.148 \\
\hline Urea $(\leq />7.5 \mathrm{mmol} / \mathrm{L})$ & $125 / 47$ & $24 / 28$ & $<0.001$ \\
\hline Creatinine $(\leq />130 \mathrm{umol} / \mathrm{L})$ & $159 / 13$ & $43 / 9$ & 0.039 \\
\hline $\mathrm{AST}(\leq />40 \mathrm{IU})$ & $96 / 55$ & $24 / 16$ & 0.678 \\
\hline
\end{tabular}


Table 3 (continued)

\begin{tabular}{|c|c|c|c|}
\hline & Alive $(n=172)$ & Dead $(n=52)$ & p-value \\
\hline $\operatorname{ALT}(\leq />56 \| U)$ & $136 / 33$ & $41 / 9$ & 0.810 \\
\hline $\operatorname{ALP}(\leq />130 \| U)$ & $155 / 14$ & $49 / 2$ & 0.294 \\
\hline Bilirubin $(\leq />17 \mathrm{mmol} / \mathrm{L})$ & $151 / 18$ & $45 / 6$ & 0.823 \\
\hline Glucose $(\leq />7 \mathrm{mmol} / \mathrm{L})$ & $96 / 51$ & $22 / 20$ & 0.128 \\
\hline Lactate $(</ \geq 2 \mathrm{mmol} / \mathrm{L})$ & $43 / 21$ & $17 / 12$ & 0.426 \\
\hline $\mathrm{HCO}_{3}(\geq /<22 \mathrm{mmol} / \mathrm{L})$ & $29 / 7$ & $14 / 4$ & 0.813 \\
\hline $\mathrm{PT}(\leq />13 \mathrm{~s})$ & $94 / 50$ & $24 / 17$ & 0.429 \\
\hline $\operatorname{APPT}(\leq />38 \mathrm{~s})$ & $133 / 8$ & $36 / 3$ & 0.642 \\
\hline
\end{tabular}

Living circumstances: $0=$ independent, $1=$ sheltered accommodation, $2=$ care home, $3=$ nursing home

Ethnicity: White $=1$, Mixed $=2$, Asian $=3$, Black $=4$, Other ethnic groups or missing $=5$

poGPS peri-operative Glasgow prognostic score, $N L R$ neutrophil lymphocyte ratio

At 30-days following admission, 52 patients had died and the mortality rate was 23\%. COVID-19 related respiratory failure $(75 \%)$ and multiorgan failure $(12 \%)$ were the commonest causes of death recorded. The relationship between 30-day mortality and clinicopathological characteristics are shown in Table 3. Death following admission for COVID-19 was associated with age $\geq 70$ years $(\mathrm{p}<0.001)$, past medical history of cognitive impairment $(\mathrm{p} \leq 0.001)$, previous delirium $(\mathrm{p}<0.001)$, clinical frailty score $>3(\mathrm{p}<0.001)$, hypertension $(p<0.05)$, heart failure $(p<0.01)$, NEWS $>4$ $(\mathrm{p}<0.01)$, positive CXR $(\mathrm{p}<0.01)$, and subsequent positive COVID-19 swab ( $\mathrm{p} \leq 0.001)$. Death was also associated with CRP $>80 \mathrm{mg} / \mathrm{L}(\mathrm{p}<0.05)$, albumin $<35 \mathrm{~g} / \mathrm{L}$ $(\mathrm{p}<0.05)$, poGPS $(\mathrm{p}<0.05)$, lymphocytes $<1.510^{9} / 1$ $(\mathrm{p}<0.05)$, neutrophil lymphocyte ratio $(\mathrm{p} \leq 0.001)$, haematocrit $(<0.40 \mathrm{~L} / \mathrm{L} \quad($ male $) /<0.37 \mathrm{~L} / \mathrm{L}$ (female) $(\mathrm{p} \leq 0.01), \quad$ urea $>7.5 \mathrm{mmol} / \mathrm{L} \quad(\mathrm{p}<0.001)$, creatinine $>130 \mathrm{mmol} / \mathrm{L}(\mathrm{p}<0.05)$ and elevated urea: albumin ratio $(<0.001)$.

To determine which admission parameters were independently associated with death within 30 days, binary logistic regression analysis was carried out (see Table 4). On analysis, age $\geq 70$ years (O.R. 3.9, 95\% C.I. $1.4-8.2, \mathrm{p}<0.001)$, past medical history of heart failure (O.R. 3.3, 95\% C.I. 1.2-19.3, p<0.05), NEWS $>4$ at presentation (O.R. 2.4, 95\% C.I. 1.1-4.4, p < 0.05), positive initial CXR (O.R. 0.4, 95\% C.I. 0.2-0.9, $\mathrm{p}<0.05$ ) and poGPS (O.R. 2.3, 95\% C.I. 1.1-4.4, p<0.05) remained independently associated with death.

Among those patients who tested PCR COVID-19 positive $(n=122)$, age $\geq 70$ years (O.R. 4.7, 95\% C.I. $2.0-11.3, \mathrm{p}<0.001)$, past medical history of heart failure (O.R. 4.4, 95\% C.I. 1.2-20.5, $\mathrm{p}<0.05$ ) and poGPS
(O.R. 2.4, 95\% C.I. 1.1-5.1, p < 0.05) remained independently associated with 30 -days mortality (see Table 5).

\section{Discussion}

The results of the present study show that, on admission and using routine clinical measures, there was a wideranging perturbance of clinicopathological parameters in patients with COVID-19. In particular, heart failure and activation of the systemic inflammatory response were independently associated with death at 30 days. Therefore, it would appear that, in addition to the current anti-viral therapeutic targets, the host systemic inflammatory response may be a legitimate therapeutic target in patients presenting to Emergency Departments with COVID-19.

The results of the present study are consistent with a number of other small studies [17-19] from Asia that have reported the usefulness of markers of the systemic inflammatory response to risk stratify patients with COVID-19. In particular, the NLR has been previously reported to have prognostic value in these patients [14, $20,21]$. For example, Liu et al. reported in a prospective validation study that NLR was a predictor of critical illness in 61 patients with PCR confirmed COVID-19 infection [21]. Similarly, Yang et al. reported in a randomly selected cohort of 69 non-severe and 24 severe cases that NLR > 3.3 was independently associated with "more severe illness" (HR 2.46; 95\% CI 1.98-4.57; p<0.05) [14]. Lagunas-Rangel reported a meta-analysis of NLR and lymphocyte-CRP ratio that included 5 studies from mainland China, comprising 828 patients, which concluded that increased NLR and low lymphocyte-CRP ratios, reflecting an enhanced inflammatory process, may suggest a poor prognosis [22]. The present study, 
Table 4 Binary logistic regression analysis of clinicopathological characteristics of patients admitted with symptoms of COVID-19 $(n=224)$

\begin{tabular}{|c|c|c|c|c|c|c|}
\hline & Alive $(n=172)$ & Dead $(n=52)$ & p-value & O.R & $95 \% \mathrm{Cl}$ & $p$-value \\
\hline Age $(</ \geq 70$ years $)$ & $124 / 48$ & $19 / 33$ & $<0.001$ & 3.9 & $1.4-8.2$ & $<0.001$ \\
\hline Sex (male/female) & $91 / 81$ & $33 / 19$ & 0.181 & & & \\
\hline $\operatorname{BMI}\left(<20 ; \geq 20-29 ; \geq 30 \mathrm{~kg} / \mathrm{m}^{2}\right)$ & $13 / 85 / 74$ & $4 / 25 / 23$ & 0.867 & & & \\
\hline SIMD (1 (most)-6 (least) deprived) & $81 / 31 / 17 / 23 / 1 / 19$ & 24/4/7/11/0/6 & 0.685 & & & \\
\hline Ethnicity $(1-5)$ & $159 / 0 / 7 / 2 / 3$ & $50 / 0 / 0 / 0 / 2$ & 0.774 & & & \\
\hline Living circumstances (0-3) & $151 / 11 / 7 / 3$ & $39 / 2 / 8 / 3$ & 0.008 & & & \\
\hline \multicolumn{7}{|l|}{ Past Medical History } \\
\hline Hypertension (y/n) & $63 / 109$ & $28 / 24$ & 0.027 & - & - & 0.229 \\
\hline Heart failure $(y / n)$ & $12 / 160$ & $11 / 41$ & 0.003 & 3.3 & $1.2-19.3$ & 0.028 \\
\hline T1DM $(y / n)$ & $2 / 170$ & $0 / 52$ & 0.436 & & & \\
\hline T2DM (y/n) & $37 / 135$ & $15 / 37$ & 0.273 & & & \\
\hline Chronic renal failure (y/n) & $18 / 154$ & $10 / 42$ & 0.095 & & & \\
\hline Cognitive impairment $(y / n)$ & $16 / 156$ & $14 / 38$ & 0.001 & & & \\
\hline Previous delirium (y/n) & $6 / 166$ & $10 / 40$ & $<0.001$ & & & \\
\hline Frailty score $(\leq />3)$ & $107 / 65$ & $14 / 38$ & $<0.001$ & & & \\
\hline $\operatorname{COPD}(\mathrm{y} / \mathrm{n})$ & 29/143 & $12 / 40$ & 0.311 & & & \\
\hline Smoker (never/ex/active) & $82 / 70 / 20$ & $24 / 27 / 1$ & 0.428 & & & \\
\hline Alcohol excess $(y / n)$ & $22 / 150$ & $9 / 43$ & 0.410 & & & \\
\hline Liver disease $(y / n)$ & $15 / 157$ & $4 / 48$ & 0.816 & & & \\
\hline Hep C (never/previous/active) & $167 / 2 / 2$ & $51 / 1 / 0$ & 0.650 & & & \\
\hline Active cancer $(\mathrm{y} / \mathrm{n})$ & $6 / 166$ & $3 / 49$ & 0.464 & & & \\
\hline \multicolumn{7}{|l|}{ Diagnostic criteria } \\
\hline PCR positive/Clinical Dx/Radiological Dx & $74 / 7 / 91$ & $37 / 1 / 14$ & 0.001 & & & \\
\hline PCR negative/indeterminate/positive & $43 / 42 / 83$ & $4 / 9 / 39$ & 0.001 & & & \\
\hline CXR negative/positive & $63 / 107$ & $30 / 21$ & 0.006 & 0.40 & $0.4-0.9$ & 0.020 \\
\hline \multicolumn{7}{|l|}{ Physiology at presentation } \\
\hline NEWS $(\leq />4)$ & $75 / 96$ & $12 / 39$ & 0.009 & 2.4 & $1.1-4.4$ & 0.024 \\
\hline Delirium (y/n) & $14 / 158$ & $12 / 36$ & 0.001 & & & \\
\hline \multicolumn{7}{|l|}{ Laboratory results at presentation } \\
\hline $\operatorname{CRP}(</ \geq 150 \mathrm{mg} / \mathrm{L})$ & $134 / 37$ & $34 / 18$ & 0.058 & & & \\
\hline Albumin $(\geq /<35 \mathrm{~g} / \mathrm{L})$ & $82 / 87$ & $15 / 35$ & 0.021 & & & \\
\hline $\operatorname{poGPS}(0 / 1 / 2)$ & $130 / 35 / 3$ & $32 / 15 / 3$ & 0.032 & 2.2 & $1.1-4.4$ & 0.024 \\
\hline $\operatorname{WCC}\left(<4.5 / \geq 4.5-\leq 11.0 />11.0 \times 10^{9} / L\right)$ & $23 / 118 / 30$ & $7 / 34 / 11$ & 0.750 & & & \\
\hline Neutrophils $\left(</ \geq 7.5 \times 10^{9} / \mathrm{L}\right)$ & $132 / 39$ & $34 / 18$ & 0.088 & & & \\
\hline Lymphocytes $\left(\geq /<1.5 \times 10^{9} / \mathrm{L}\right)$ & $52 / 118$ & $7 / 45$ & 0.015 & & & \\
\hline $\operatorname{NLR}(<3 / 3-5 / \geq 5)$ & $39 / 46 / 85$ & $5 / 9 / 38$ & 0.004 & - & - & 0.126 \\
\hline $\mathrm{Hb}(\geq /<12.0 \mathrm{~g} / \mathrm{dL})$ & $143 / 28$ & $38 / 13$ & 0.142 & & & \\
\hline $\mathrm{MCV}(>/ \leq 99 \mathrm{fl})$ & $149 / 21$ & $40 / 11$ & 0.102 & & & \\
\hline Hct (male $\geq /<0.40$ ) (female $\geq /<0.37$ ) L/L & $126 / 45$ & $29 / 23$ & 0.014 & - & - & 0.125 \\
\hline Platelets $\left(\geq /<150 \times 10^{9}\right)$ & $140 / 30$ & $40 / 12$ & 0.383 & & & \\
\hline Sodium $(<133 / \geq 133-\leq 146 />146 \mathrm{mmol} / \mathrm{L})$ & $17 / 153 / 2$ & $9 / 40 / 3$ & 0.013 & & & \\
\hline Potassium $(<3.5 / \geq 3.5-\leq 5.5 />5.5 \mathrm{mmol} / \mathrm{L})$ & $13 / 142 / 2$ & $5 / 36 / 0$ & 0.822 & & & \\
\hline $\mathrm{Mg}(\geq /<0.75 \mathrm{mmol} / \mathrm{L})$ & $33 / 60$ & $8 / 28$ & 0.148 & & & \\
\hline Urea $(\leq />7.5 \mathrm{mmol} / \mathrm{L})$ & $125 / 47$ & $24 / 28$ & $<0.001$ & & & \\
\hline Creatinine $(\leq />130 \mathrm{umol} / \mathrm{L})$ & $159 / 13$ & $43 / 9$ & 0.039 & & & \\
\hline $\mathrm{AST}(\leq />40 \mathrm{IU})$ & $96 / 55$ & $24 / 16$ & 0.678 & & & \\
\hline $\operatorname{ALT}(\leq />56 \mathrm{IU})$ & $136 / 33$ & $41 / 9$ & 0.810 & & & \\
\hline
\end{tabular}


Table 4 (continued)

\begin{tabular}{|c|c|c|c|c|c|c|}
\hline & Alive $(n=172)$ & Dead $(n=52)$ & p-value & O.R & $95 \% \mathrm{Cl}$ & p-value \\
\hline $\operatorname{ALP}(\leq />130$ IU $)$ & $155 / 14$ & $49 / 2$ & 0.294 & & & \\
\hline Bilirubin $(\leq />17 \mathrm{mmol} / \mathrm{L})$ & $151 / 18$ & $45 / 6$ & 0.823 & & & \\
\hline
\end{tabular}

Living circumstances: $0=$ independent, $1=$ sheltered accommodation, $2=$ care home, $3=$ nursing home

Ethnicity: White $=1$, Mixed $=2$, Asian $=3$, Black $=4$, Other ethnic groups $=5$

poGPS peri-operative Glasgow prognostic score, NLR neutrophil lymphocyte ratio

compared with these previous studies, has greater detail in the admission clinicopathological characteristics, thereby reducing the potential confounding of unmeasured factors. Moreover, it uses a standardised measure of the systemic inflammatory response (developed to assess the magnitude of the post-operative systemic inflammatory response)-the poGPS [6]. This study is also among the first to provide data from a U.K. population.

Data from the present study are also consistent with recent reports of an inverse relationship between age and BMI among patients with COVID-19 who were admitted to the intensive care setting (see Fig. 1) [23]. However, contrary to recent reports [24, 25], no significant association was found between BMI and mortality in the present study. Interestingly, a BMI of $40 \mathrm{~kg} / \mathrm{m}^{2}$ or higher was identified by the Centre for Disease Control and Prevention in the USA as a factor that may increase a person's vulnerability to severe COVID-19 infection [26]. Nonetheless, despite the association between a BMI in the obese range and established risk factors for severe COVID-19 infection, such as type 2 diabetes, hypertension, and cardiovascular disease [25], data from the present study do not support an association with an increase in COVID-19 related mortality. The reasons for this are not clear. However, the present sample size may not have been large enough to detect the effect of obesity. Nevertheless, obesity compared with other physiological and inflammatory parameters is likely to have a smaller effect and may not provide a useful therapeutic target.

The post-operative systemic inflammatory response and the subsequent metabolic stress has been the subject of continuing interest over the last 40 years. In particular, there are well-developed therapeutic strategies to moderate the systemic inflammatory response. These include minimising surgical trauma and psychological distress; the use of anti-inflammatory agents (steroids) and antibiotics; fluid optimisation; optimal glucose control and nutritional support, to form an enhanced recovery strategy [7]. If the present results are confirmed, then it may be that using this knowledge to moderate the systemic inflammatory response associated with COVID-19 may reduce mortality. Indeed, the role of dexamethasone is endorsed by the recent positive report from the RECOVERY trial that showed a significant survival benefit at 28-days among patients who required either invasive mechanical ventilation or oxygen alone at randomization but not among those receiving no respiratory support [27]. Furthermore, there is emerging evidence of the importance of pro-inflammatory cytokines interleukin-6 (IL-6) and tumour necrosis factor (TNF-alpha) as predictors of mortality in patients with COVID-19 [28] and that IL-6 blockade appears to be beneficial [29]. Biran et al.have recently reported in a retrospective multi-centre observational study of 764 patients with severe SARS-CoV-2 infection requiring ICU support that treatment with a recombinant monoclonal antibody against the interleukin (IL)-6 receptor, Tocilizumab ${ }^{\circledR}$, was associated with a $25 \%$ reduction in hospital-related mortality [29]. Randomised trials are required to confirm these results.

Despite the massive surge in COVID-19 related deaths, a relatively small proportion of the overall number infected have become unwell [1]. However, among those who do become unwell, clinical deterioration due to cytokine storm can occur with alarming rapidity, and mortality is high $[18,30]$. The present results are consistent with these results and importantly offer a means of routine clinical assessment of an on-going systemic inflammatory response and its treatment since proinflammatory cytokine measurements are not routinely available from clinical laboratories.

Numerous randomised controlled trails of anti-viral agents are ongoing based on the premise that treating viral infection may benefit patients by reducing viral load and aiding recovery. To date, none of these studies have reported a significant mortality benefit. Therefore, in the absence of such direct intervention it may be important to minimise the systemic inflammatory response and support host metabolism in line with optimal peri-operative care. This strategy has the advantage of being part of routine clinical care and may complement more aggressive anti-viral strategies.

The present study has a number of limitations. There was a relatively small sample size and therefore subject to limitations such as sample bias. In addition, the ethnic background of the patients within this study was 
Table 5 Binary logistic regression analysis of clinicopathological characteristics of patients admitted who had COVID-19 $P C R+(n=122)$

\begin{tabular}{|c|c|c|c|c|c|c|}
\hline & Alive $(n=83)$ & Dead $(n=39)$ & p-value & O.R & $95 \% \mathrm{Cl}$ & $p$-value \\
\hline Age $(</ \geq 70$ years $)$ & $53 / 30$ & $12 / 27$ & $<0.001$ & 4.7 & $2.0-11.3$ & 0.001 \\
\hline Sex (male/female) & $39 / 44$ & $16 / 23$ & 0.219 & & & \\
\hline $\operatorname{BMI}\left(<20 ; \geq 20-29 ; \geq 30 \mathrm{~kg} / \mathrm{m}^{2}\right)$ & $5 / 37 / 41$ & $3 / 18 / 18$ & 0.798 & & & \\
\hline SIMD (1 (least)-6 (most) deprived) & $9 / 1 / 16 / 10 / 21 / 37$ & $4 / 0 / 9 / 6 / 2 / 21$ & 0.959 & & & \\
\hline Ethnicity $(1-5)$ & $79 / 0 / 3 / 1 / 0$ & $37 / 0 / 0 / 0 / 2$ & 0.441 & & & \\
\hline Living circumstances (0-3) & $72 / 8 / 2 / 1$ & $30 / 0 / 7 / 2$ & 0.027 & & & \\
\hline \multicolumn{7}{|l|}{ Past Medical History } \\
\hline Hypertension (y/n) & $33 / 50$ & $20 / 19$ & 0.233 & - & - & 0.765 \\
\hline Heart failure (y/n) & $4 / 79$ & $7 / 32$ & 0.019 & 4.4 & $1.1-18.6$ & 0.042 \\
\hline $\operatorname{T1DM}(y / n)$ & $1 / 82$ & $0 / 39$ & 0.493 & & & \\
\hline $\mathrm{T} 2 \mathrm{DM}(\mathrm{y} / \mathrm{n})$ & $22 / 61$ & $10 / 29$ & 0.920 & & & \\
\hline Chronic renal failure $(y / n)$ & $9 / 74$ & $8 / 31$ & 0.152 & & & \\
\hline Cognitive impairment (y/n) & $8 / 75$ & $12 / 27$ & 0.003 & & & \\
\hline Previous delirium (y/n) & $3 / 80$ & $10 / 28$ & $<0.001$ & & & \\
\hline Frailty score $(\leq />3)$ & $53 / 30$ & $8 / 31$ & $<0.001$ & & & \\
\hline $\operatorname{COPD}(y / n)$ & $11 / 72$ & $11 / 28$ & 0.046 & - & - & 0.279 \\
\hline Smoker & $41 / 35 / 7$ & $16 / 22 / 1$ & 0.833 & & & \\
\hline Alcohol excess (y/n) & $5 / 78$ & $5 / 34$ & 0.204 & & & \\
\hline Liver disease $(y / n)$ & $6 / 77$ & $2 / 37$ & 0.663 & & & \\
\hline Hep C (never/previous/active) & $82 / 0 / 1$ & $39 / 0 / 0$ & 0.493 & & & \\
\hline Active cancer (y/n) & $4 / 79$ & $1 / 38$ & 0.560 & & & \\
\hline \multicolumn{7}{|l|}{ Diagnostic criteria } \\
\hline CXR negative/positive & $38 / 45$ & $22 / 16$ & 0.218 & & & \\
\hline \multicolumn{7}{|l|}{ Physiology at presentation } \\
\hline NEWS $(\leq />4)$ & $36 / 46$ & $10 / 29$ & 0.054 & - & - & 0.146 \\
\hline Delirium $(y / n)$ & $6 / 77$ & $10 / 27$ & 0.003 & & & \\
\hline \multicolumn{7}{|l|}{ Laboratory results at presentation } \\
\hline $\operatorname{poGPS}(0 / 1 / 2)$ & $63 / 16 / 2$ & $24 / 11 / 3$ & 0.063 & 2.4 & $1.1-5.1$ & 0.027 \\
\hline $\operatorname{NLR}(<3 / 3-5 / \geq 5)$ & $21 / 23 / 38$ & $5 / 6 / 28$ & 0.015 & - & - & 0.144 \\
\hline $\mathrm{Hb}(\geq /<12.0 \mathrm{~g} / \mathrm{dL})$ & $65 / 17$ & $28 / 10$ & 0.497 & & & \\
\hline $\mathrm{MCV}(\leq />99 \mathrm{fl})$ & $75 / 7$ & $30 / 9$ & 0.028 & - & - & 0.235 \\
\hline $\operatorname{Hct}($ male $\geq /<0.40)$ (female $\geq /<0.37) \mathrm{L} / \mathrm{L}$ & $59 / 23$ & $23 / 16$ & 0.155 & & & \\
\hline Platelets $\left(<150 / \geq 150-<450 / \geq 450 \times 10^{9}\right)$ & $14 / 67 / 0$ & 9/30/1 & 0.452 & & & \\
\hline Sodium ( $\leq 133 / 134-145 / \geq 146 \mathrm{mmol} / \mathrm{L})$ & 10/71/2 & $5 / 31 / 3$ & 0.240 & & & \\
\hline Potassium $(<3.5 / \geq 3.5-\leq 5.3 />53 \mathrm{mmol} / \mathrm{L})$ & $5 / 73 / 0$ & $3 / 27 / 0$ & 0.525 & & & \\
\hline $\mathrm{Mg}(\geq /<0.75 \mathrm{mmol} / \mathrm{L})$ & $12 / 3$ & $4 / 2$ & 0.527 & & & \\
\hline Urea $(\leq />7.0 \mathrm{mmol} / \mathrm{L})$ & $57 / 26$ & $17 / 22$ & 0.008 & & & \\
\hline Creatinine $(\leq />130$ umol/L) & $76 / 7$ & $34 / 5$ & 0.450 & & & \\
\hline $\operatorname{AST}(\leq />40 \mathrm{IU})$ & $48 / 26$ & $21 / 28$ & 0.466 & & & \\
\hline $\operatorname{ALT}(\leq />56 \mathrm{IU})$ & $69 / 12$ & $33 / 5$ & 0.810 & & & \\
\hline $\operatorname{ALP}(\leq />150 \mid U)$ & $75 / 6$ & $37 / 1$ & 0.304 & & & \\
\hline Bilirubin ( $\leq />17 \mathrm{mmol} / \mathrm{L})$ & $78 / 3$ & $35 / 3$ & 0.332 & & & \\
\hline Glucose $(\leq />7 \mathrm{mmol} / \mathrm{L})$ & $40 / 27$ & $18 / 12$ & 0.978 & & & \\
\hline Lactate $(</ \geq 2 \mathrm{mmol} / \mathrm{L})$ & $24 / 6$ & $13 / 7$ & 0.241 & & & \\
\hline $\mathrm{HCO}_{3}(\geq /<22 \mathrm{mmol} / \mathrm{L})$ & $16 / 4$ & $9 / 3$ & 0.744 & & & \\
\hline $\mathrm{PT}(\leq />13 \mathrm{~s})$ & $53 / 16$ & $19 / 11$ & 0.169 & & & \\
\hline $\operatorname{APPT}(\leq />38 \mathrm{~s})$ & $67 / 3$ & $3 / 26$ & 0.253 & & & \\
\hline
\end{tabular}

Living circumstances: $0=$ independent, $1=$ sheltered accommodation, $2=$ care home, $3=$ nursing home Ethnicity: White $=1$, Mixed $=2$, Asian $=3$, Black $=4$, Other ethnic groups $=5$

poGPS peri-operative Glasgow prognostic score, NLR neutrophil lymphocyte ratio 


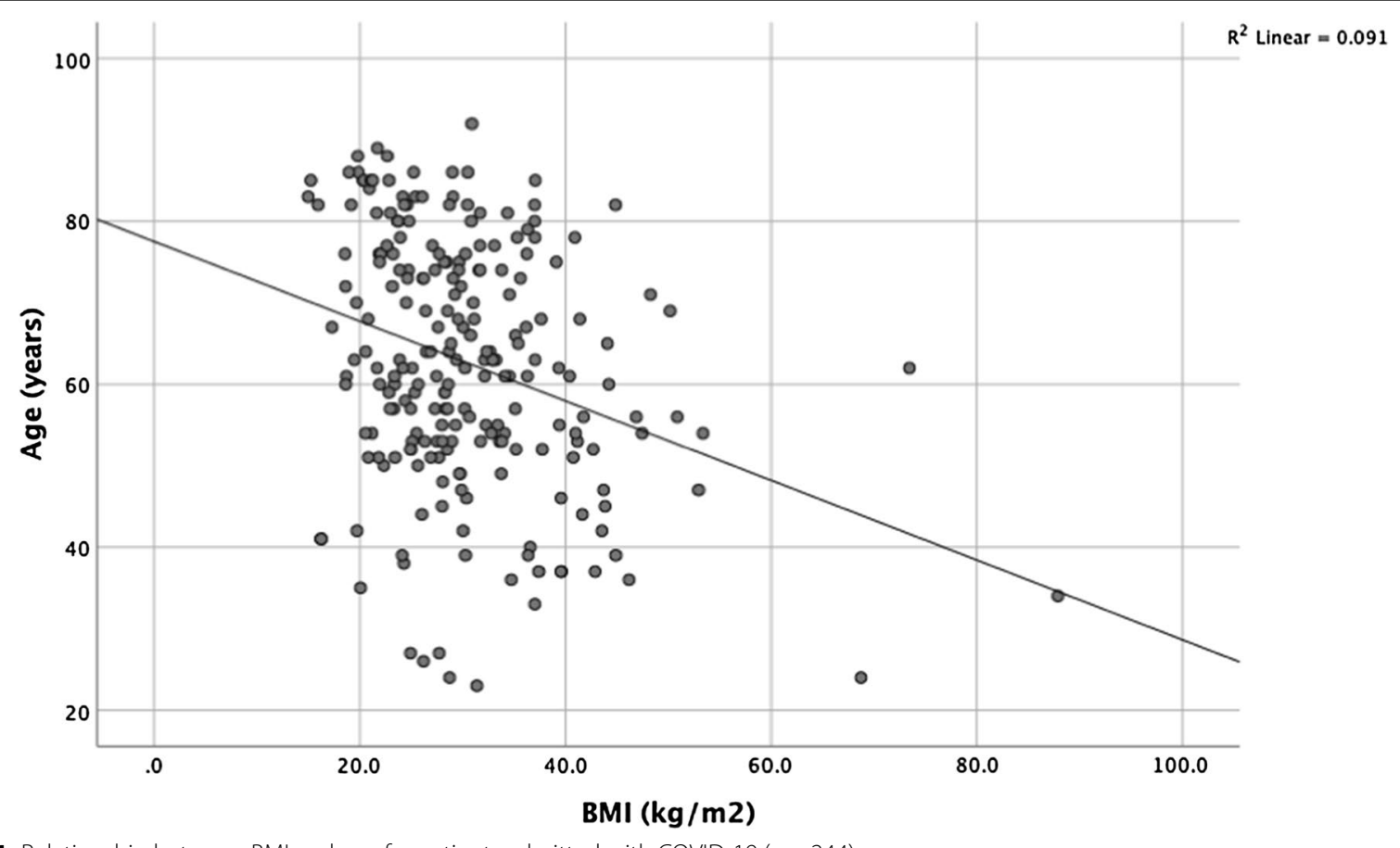

Fig. 1 Relationship between BMl and age for patients admitted with COVID-19 $(n=244)$

not as diverse as in other population centres globally. Furthermore, the level of deprivation was relatively high compared to other population centres in the UK. However, this study was based on routine clinical measurements and therefore readily subject to confirmation. Other less commonly utilised measurements, such as LDH and D-dimer have also been reported to have prognostic value in patients with COVID-19 infection [2]. However, these are recognised to be elevated as part of the systemic inflammatory response and may reflect the same process. Therefore, further work is required to rationalise the use of markers of the systemic inflammatory response in patients with COVID-19.

\section{Conclusion}

Old age and severe systemic inflammation, as evidenced by the post-operative Glasgow Prognostic Score (poGPS), were independently associated with 30-day mortality in patients admitted to hospital with COVID19 infection.

\footnotetext{
Abbreviations

COVID-19: Novel corona virus-19; SIRS: Systemic inflammatory response; NEWS: National early warning score; PCR: Polymerase chain reaction; poGPS: Peri-operative Glasgow Prognostic Score; NLR: Neutrophil lymphocyte ratio; CRP: Serum C-reactive protein; GRI: Glasgow Royal Infirmary; ED: Emergency Department; AAU: Acute Assessment Unit; CXR: Chest X-ray; BMI: Body mass index; CFS: Clinical Frailty Scale; AVPU scale: Alert (A), Responding to verbal
}

(V), painful (P) stimuli and unresponsive (U); SIMD: Scottish Indices of Multiple Deprivation; $x^{2}$ test: Chi-squared test.

\section{Acknowledgements}

The research team wish to acknowledge the assistance of Mrs. Jill Dempster (Project Management Unit, Research and Development Department, Greater Glasgow and Clyde) for her expertise and dedication in relation to this work.

\section{Authors' contributions}

$\mathrm{DM}, \mathrm{DCMM}, \mathrm{RD}, \mathrm{DT}$ and $\mathrm{BL}$ conceived the idea for the study. DM, DCMM, RD, $D T, I G, A D$ and $B L$ contributed to the study design. MW, CR, JWV, WMS, OEK, DCM, SLR, LDB and NG performed manual data extraction from the electronic patient records. AC performed post-code analysis and deprivation scoring. All authors read and approved the final manuscript.

\section{Funding}

DT is funded by the Scottish Trace Elements and Micronutrients Diagnostic and Reference Laboratory. DMcM is funded by the University of Glasgow.

\section{Availability of data and materials}

Anonymized data will be made available on request to the corresponding author.

\section{Ethics approval and consent to participate}

This study was approved by the NHS Greater Glasgow and Clyde Caldicott guardian. The study protocol (GN20AE307) was approved by the North West England - Preston research ethics committee (20/NW/0336) and registered with clinicaltrials.gov (NCT04484545). Patient consent to participate was waived.

\section{Consent for publication}

All authors have consented to publication and are guarantors of the manuscript and data presented.

\section{Competing interests}

None of the authors have any conflict of interest to declare. 


\begin{abstract}
Author details
${ }^{1}$ Emergency Medicine Department, Glasgow Royal Infirmary, 84 Castle Street, Glasgow G4 OSF, UK. ${ }^{2}$ School of Medicine Veterinary and Life Sciences, Wolfson Medical School Building, University of Glasgow, University Avenue, Glasgow G12 8QQ, UK. ${ }^{3}$ Academic Unit of Surgery, School of Medicine, University of Glasgow, New Lister Building, Royal Infirmary, Glasgow G31 2ER, UK. ${ }^{4}$ Department of Acute Medicine, Glasgow Royal Infirmary, Glasgow G4 OSF, UK. ${ }^{5}$ Institute of Genetics and Molecular Medicine, University of Edinburgh, Edinburgh EH4 2XU, UK. ${ }^{6}$ St Columba's Hospice, 15 Boswall Rd, Edinburgh EH5 3RW, UK. ${ }^{7}$ The Scottish Trace Element and Micronutrient Reference Laboratory, Department of Biochemistry, Royal Infirmary, Glasgow G31 2ER, UK. ${ }^{8}$ Department of Clinical Biochemistry, Queen Elizabeth University Hospital, Govan, Glasgow G51 4TF, UK.
\end{abstract}

Received: 6 July 2020 Accepted: 8 September 2020 Published online: 15 September 2020

\section{References}

1. 2020. WC-cpM. https://www.worldometers.info/coronavirus/.

2. Zhang ZL, Hou YL, Li DT, Li FZ. Laboratory findings of COVID-19: a systematic review and meta-analysis. Scand J Clin Lab Invest. 2020;1:1-7.

3. Tian W, Jiang W, Yao J, Nicholson CJ, Li RH, Sigurslid HH, et al. Predictors of mortality in hospitalized COVID-19 patients: a systematic review and meta-analysis. J Med Virol. 2020. https://doi.org/10.1002/jmv.26050.

4. Hotamisligil GS. Foundations of Immunometabolism and Implications for Metabolic Health and Disease. Immunity. 2017;47(3):406-20.

5. Gabay C, Kushner I. Acute-phase proteins and other systemic responses to inflammation. New Engl J Med. 1999;340(6):448-54.

6. Watt DG, McSorley ST, Park JH, Horgan PG, McMillan DC. A Postoperative systemic inflammation score predicts short- and long-term outcomes in patients undergoing surgery for colorectal cancer. Ann Surg Oncol. 2017;24(4):1100-9.

7. Watt DG, McSorley ST, Horgan PG, McMillan DC. Enhanced Recovery after surgery: which components, if any, impact on the systemic inflammatory response following colorectal surgery?: a systematic review. Medicine (Baltimore). 2015;94(36):e1286.

8. Lobo DN, Gianotti L, Adiamah A, Barazzoni R, Deutz NEP, Dhatariya K, et al. Perioperative nutrition: recommendations from the ESPEN expert group. Clin Nutr. 2020. https://doi.org/10.1016/j.clnu.2020.03.038.

9. Onwochei DN, Fabes J, Walker D, Kumar G, Moonesinghe SR. Critical care after major surgery: a systematic review of risk factors for unplanned admission. Anaesthesia. 2020;75(Suppl 1):e62-e74.

10. Health Protection Scotland (Government Document). https://hpspubsrep o.blob.core.windows.net/hps-website/nss/2936/documents/1_covid -19-guidance-for-secondary-care.pdf.

11. Keep JW, Messmer AS, Sladden R, Burrell N, Pinate R, Tunnicliff M, et al. National early warning score at Emergency Department triage may allow earlier identification of patients with severe sepsis and septic shock: a retrospective observational study. Emerg Med J. 2016;33(1):37-41.

12. Rockwood K, Song X, MacKnight C, Bergman H, Hogan DB, McDowell I, et al. A global clinical measure of fitness and frailty in elderly people. CMAJ. 2005;173(5):489-95.

13. Wallis SJ, Wall J, Biram RW, Romero-Ortuno R. Association of the clinical frailty scale with hospital outcomes. QJM. 2015;108(12):943-9.

14. Yang AP, Liu JP, Tao WQ, Li HM. The diagnostic and predictive role of NLR, d-NLR and PLR in COVID-19 patients. Int Immunopharmacol. 2020;84:106504.

15. Dolan RD, Laird BJA, Horgan PG, McMillan DC. The prognostic value of the systemic inflammatory response in randomised clinical trials in cancer: A systematic review. Critical Reviews in Oncology / Hematology. 2018;132:130-7.

16. Dolan RD, McSorley ST, Park JH, Watt DG, Roxburgh CS, Horgan PG, et al The prognostic value of systemic inflammation in patients undergoing surgery for colon cancer: comparison of composite ratios and cumulative scores. Br J Cancer. 2018;119(1):40-51.

17. Zhu W, Xie K, Lu H, Xu L, Zhou S, Fang S. Initial clinical features of suspected coronavirus disease 2019 in two emergency departments outside of Hubei, China. J Med Virol. 2020.

18. Chen T, Wu D, Chen H, Yan W, Yang D, Chen G, et al. Clinical characteristics of 113 deceased patients with coronavirus disease 2019: retrospective study. BMJ. 2020;368:m1091.

19. Mehta P, McAuley DF, Brown M, Sanchez E, Tattersall RS, Manson JJ, et al. COVID-19: consider cytokine storm syndromes and immunosuppression. Lancet. 2020;395(10229):1033-4.

20. Qin C, Zhou L, Hu Z, Zhang S, Yang S, Tao Y, et al. Dysregulation of immune response in patients with COVID-19 in Wuhan. China. Clin Infect Dis. 2020;71(15):762-8. https://doi.org/10.1093/cid/ciaa248.

21. Liu J, Liu Y, Xiang P, Pu L, Xiong H, Li C, et al. Neutrophil-to-lymphocyte ratio predicts critical illness patients with 2019 coronavirus disease in the early stage. J Transl Med. 2020;18(1):206.

22. Lagunas-Rangel FA. Neutrophil-to-lymphocyte ratio and lymphocyte-toC-reactive protein ratio in patients with severe coronavirus disease 2019 (COVID-19): A meta-analysis. J Med Virol. 2020. https://doi.org/10.1002/ jmv.25819.

23. Kass DA, Duggal P, Cingolani O. Obesity could shift severe COVID-19 disease to younger ages. Lancet. 2020;395(10236):1544-5.

24. Caussy C, Wallet F, Laville M, Disse E. Obesity is Associated with Severe Forms of COVID-19. Obesity (Silver Spring). 2020.

25. Stefan N, Birkenfeld AL, Schulze MB, Ludwig DS. Obesity and impaired metabolic health in patients with COVID-19. Nat Rev Endocrinol. 2020. https://doi.org/10.1038/s41574-020-0364-6.

26. Flint SW, Tahrani AA. COVID-19 and obesity-lack of clarity, guidance, and implications for care. Lancet Diabetes Endocrinol. 2020;8(6):474-5.

27. Horby P, Lim WS, Emberson JR, Mafham M, Bell JL, Linsell L, et al. Dexamethasone in hospitalized patients with COVID-19 - preliminary report. N Engl J Med. 2020. https://doi.org/10.1056/NEJMoa2021436.

28. Del Valle DM, Kim-Schulze S, Huang HH, Beckmann ND, Nirenberg S, Wang B, et al. An inflammatory cytokine signature predicts COVID-19 severity and survival. Nat Med. 2020. https://doi.org/10.1038/s4159 1-020-1051-9.

29. Biran N, Ip A, Ahn J, Go RC, Wang S, Mathura S, et al. Tocilizumab among patients with COVID-19 in the intensive care unit: a multicentre observational study. Lancet Rheumatol. 2020. https://doi.org/10.1016/S2665 -9913(20)30277-0.

30. Goh KJ, Choong MC, Cheong EH, Kalimuddin S, Duu Wen S, Phua GC, et al. Rapid progression to acute respiratory distress syndrome: review of current understanding of critical illness from COVID-19 Infection. Ann Acad Med Singapore. 2020;49(1):1-9.

\section{Publisher's Note}

Springer Nature remains neutral with regard to jurisdictional claims in published maps and institutional affiliations.

Ready to submit your research? Choose BMC and benefit from:

- fast, convenient online submission

- thorough peer review by experienced researchers in your field

- rapid publication on acceptance

- support for research data, including large and complex data types

- gold Open Access which fosters wider collaboration and increased citations

- maximum visibility for your research: over $100 \mathrm{M}$ website views per year

At BMC, research is always in progress.

Learn more biomedcentral.com/submissions 